\title{
A Novel Robotic Arm for Tactile Perception Using Tele- Operation and Haptic Feedback
}

\author{
Vrinda Anand \\ Amity International School Vasundhara Sector 1, Ghaziabad, Uttar Pradesh, 201011, INDIA; vrinda.naomi0406@gmail.com
}

ABSTRACT: In this research, a cost-effective robotic arm was designed and developed, which empowers a user to clasp
objects with a third arm and give a sense of touch corresponding to the force that an effector encounters. A cost-effective tele-
operated robotic arm was intended to provide surgeons with tactile perception, as well as handle nuclear waste and biohazards.
Tele-operation and haptic feedback was achieved with infrared (IR) sensors, servo motors, and force pressure sensors. This arm
is capable of lifting $600 \mathrm{~g}$ which can be further increased by using pneumatic actuators. The robot could also assist patients with
weak arm strength (Amyotrophic lateral sclerosis) and stroke patients as their movements can be controlled by the user's facial
expressions.

KEYWORDS: Engineering Mechanics; Mechanical Engineering; Robotic arm; Effector; Tactile, Haptic feedback; Teleoperated robotic arm; Sensors.

\section{Introduction}

Robots have been proven an efficient workhorse for automotive and other manufacturing industries. In comparison, a robotic arm is a mechanical version of the human arm that is programmable and can perform functions that humans cannot achieve. ${ }^{1}$ Such arms can solve many limitations that humans face. The human arm is notable for its ability to perform a great variety of tasks. Although slower, weaker, and less accurate than high-performance robots of today, the human arm is without equal in terms of versatility, robustness and gracefulness. It can help humans to be self-dependent and not rely on others for their daily chores. ${ }^{2} \mathrm{~A}$ robotic arm can be designed to perform any desired task, such as welding, gripping, spinning, etc., depending on the required application. ${ }^{3}$ Robots and their components have evolved significantly to be more capable and versatile, but there still exists much room to explore. ${ }^{4,5}$

This research aimed to design and develop a cost-effective robotic arm. This includes a robotic arm, smart glasses (facial expression detection), and pneumatic muscles. Haptic technology was used to provide a sense of touch, similar to the technology used by surgeons during robot-assisted surgery.

The idea was to replace the expensive technology with an affordable and stable technology that could perform diverse functions. With a novel tactile perception method, it is possible to provide a sense of touch to the users with force pressure sensors exactly equivalent to the force being experienced by the effector. A brain, artificial intelligence (AI), was deliberately not given to the robotic arm to keep it as a tool and designed it in such a way that these are entirely dependent on user inputs.

Further, the McKibben muscles were also included, which could be used as a cheaper version of the arm's actuators. A McKibben muscle is an actuator which converts pneumatic (or hydraulic) energy into mechanical form by transferring the pressure applied on the inner surface of its bladder into the shortening tension. ${ }^{6}$

\section{Materials and Methods}

The robotic arm consisted of metal, wood, plastic, servo motors, Arduino UNO ATmega 328P, IR Obstacle Sensor Module, Thin Film Force pressure sensors and other low-cost materials.

\section{Servo Motors:}

Servo: Detects the operation error of a mechanism, provides feedback and corrects faults. A servo (Figure 1) motor can have alternating current (AC), direct current (DC) or stepper motors. Servo motors are the kinds of motors that can fulfill the commands wanted. They can operate steadily even at very small or very large speeds. A set of servos controlled the wrist and the elbow movement. A micro servo was positioned on the wrist part and a high torque servo on the elbow part.

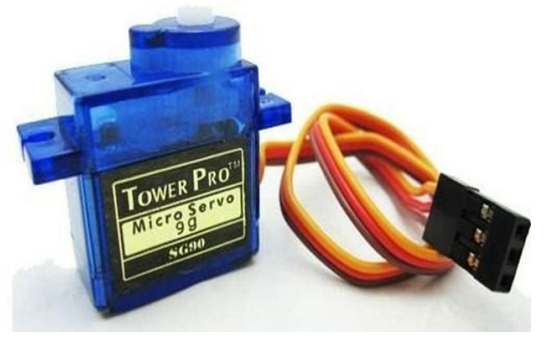

Figure 1: Servo motor.

\section{Force Pressure Sensor:}

Force Sensing Resistors (FSR) are a polymer thick film (PTF) device (Figure 2) which exhibits a decrease in resistance with an increase in the force applied to the active surface. Its force sensitivity is optimized for use in human touch control of electronic devices. This robotic arm was equipped with a sense of touch capability which was achieved by force pressure sensors. Force pressure sensors are placed on the fingertip of the robotic arm and connected to a servo motor with an Arduino Uno to provide haptic technology to the arm. 


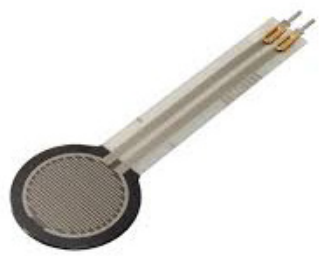

Figure 2: Force pressure sensor.

\section{Arduino Uno:}

Arduino/Genuino Uno (Figure 3) is a microcontroller board based on the ATmega328P (datasheet). It has 14 digital input/output pins (of which 6 can be used as PWM outputs), 6 analog inputs, a $16 \mathrm{MHz}$ quartz crystal, a USB connection, a power jack, an ICSP header and a reset button.



Figure 3: Force pressure sensor.

\section{Infrared Sensors:}

An infrared sensor (Figure 4) is an electronic device that emits light in order to sense some aspects of the surroundings. An IR sensor can measure the heat of an object as well as detects the motion. Some infrared sensors were added to a pair of eyeglasses and a controller glove for the robot's tele-operation. These were then attached to the servo motors through an Arduino Uno. The infrared sensor, attached to the front part of the eyeglasses, detects the closing eyelid motion, and sends the signal to the servos, which then moves the fingers of the robotic hand.

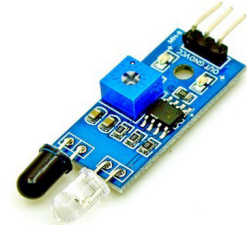

Figure 4: Force pressure sensor.

\section{Circuit Diagrams:}

For Tele-operation (Figure 5)

In order to achieve tele-operation, connect the IR sensors, servo motors and Arduino board as shown in the circuit (Figure 5).

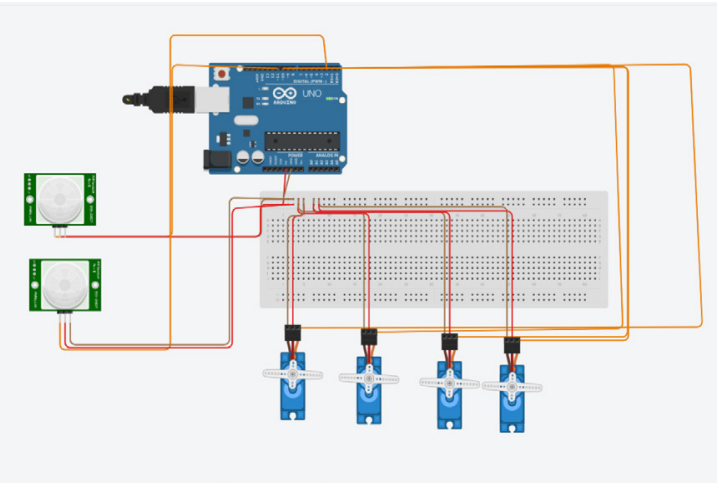

Figure 5: Infrared sensors and servos connected.

\section{Haptic Technology (Figure 6):}

For haptic technology, connect a force pressure sensor, servo motor and Arduino board together with a resistor of 10 Kohm.

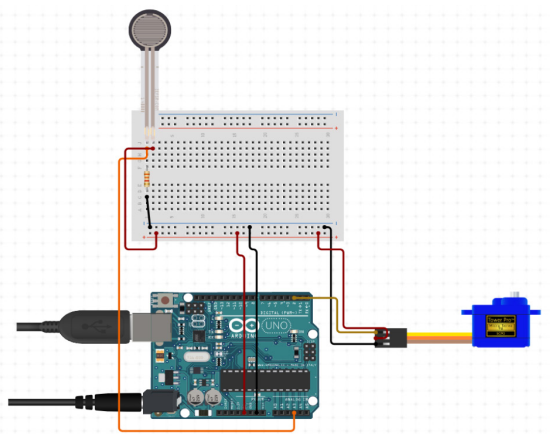

Figure 6: Force pressure sensor and servo connected.

Toque Calculation:

Forearm Torque Calculation:

Length $=23 \mathrm{~cm}$, Mass $=0.06 \mathrm{~kg}$

(Where $\mathrm{T}$ is Torque, $\mathrm{F}$ is force and $\mathrm{S}$ is the length of the forearm) (Figure 7)

$\mathrm{T}=\mathrm{F}^{*} \mathrm{~S}$

$=23^{*} 0.06 * 1 / 2$ (considering center of mass)

$\approx 0.7 \mathrm{kgcm}$
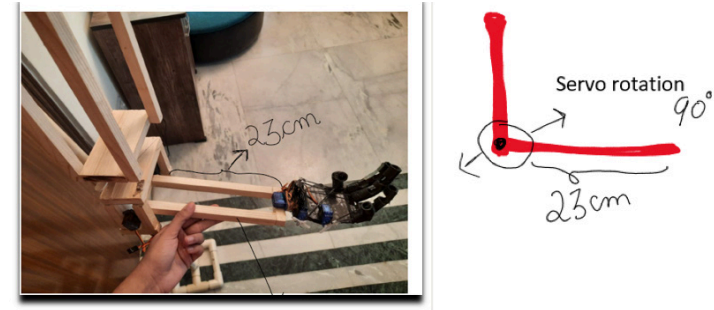

Figure 7: The forearm.

We have used a $15 \mathrm{~kg}-\mathrm{cm}$ torque servo motor.

NOTE: The torque calculated is only of the forearm.

On Adding Weight (Figure 8)

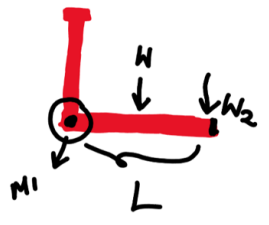

Figure 8: On adding weight on the arm.

$\mathrm{M} 1=\mathrm{S}^{*} 1 / 2 * \mathrm{~W} 1+\mathrm{S} * \mathrm{~W} 2$

$=23^{*} 1 / 2 * 0.06+23^{*} \mathrm{~W} 2$

$=0.7+23 \mathrm{~W} 2$

As the motor (M1) used has $15 \mathrm{~kg} \mathrm{~cm}$ of torque (T)

$\mathrm{W} 2=600 \mathrm{~g}$

Hence, the weight that this forearm can carry is $600 \mathrm{~g}$.

\section{Results and Discussion \\ Robotic Arm:}

The robotic arm has $3 \mathrm{D}$ rotation capability. The wrist revolves 180 degrees, 90 degrees of elbow movement, and 360 degrees of bicep rotation (Figure 9). The features ranged from tele-operation to sense of touch. Innovative methods that are quite affordable were used to give this arm such features. A 
detailed description of the features, capabilities, and intended uses is provided in the following sections.



Figure 9: The robotic arm.

\section{Tele-operation:}

Tele-operation means operating a machine at a distance. This robot was tele-operated to make the robot copy users' hand movements, which requires correct sensors to be used. Initially, flex sensors were used which were working nicely, but the cost and the durability were a concerning factor. With each sensor costing around $\$ 7$, the project's overall cost was too high.

Instead, the sensors were replaced with self-made ones, as shown in (Figure 10). These sensors were made with cardboard, graphite, and some aluminum foil. These flex sensors were working well with the servo motors. However, the challenge was its consistency. The sensors stopped working after a month, which led to a search for sensors that could work with the servos. A variety of sensors were found that exists today, including Brainwave sensors.

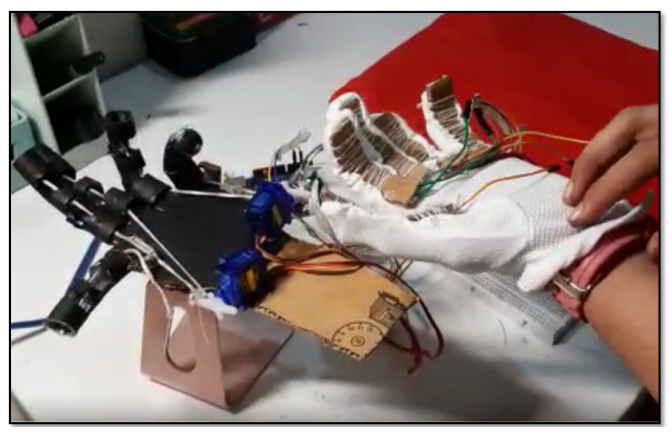

Figure 10: Homemade flex sensors attached to the servos.

One can control anything just with the power of your brain; the power that humans have always wanted has now been achieved by some great minds. ${ }^{5}$ Not only Brainwave but also the EMG sensors were pretty eye-catching. The only problem with these sensors is that they are exorbitantly priced. Hence, the reason these sensors were not used in this research.

Finally, it was observed that the infrared sensors were working well with the servos. They were coordinating perfectly with the servo motors. Not just that, but they were economically friendly too, about $\$ 1$. So, it was decided to go ahead with these sensors for this project. They were placed inside the controller glove of the left hand under the fingers (Figure 11). After the sensor senses the motion of bending fingers, each servo attached to a robotic finger, rotates, and controls the mechanical fingers as it pulls and releases them (closing and opening of the fingers).

\section{Sense of Touch:}

Haptic technology, also known as kinaesthetic communication or 3D touch, refers to any technology that can create an experience of contact by applying force, vibrations, or motions to the user.
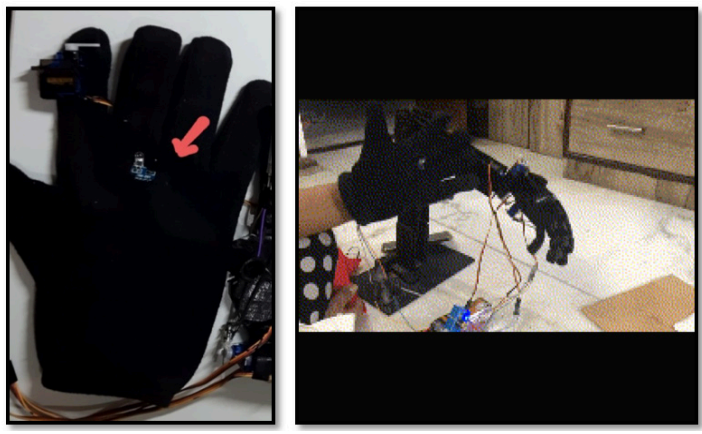

Figure 11: Controller glove with infrared sensors.

There is a force pressure attached to the fingertips of the robotic hand, which upon touching, sends those signals to the servo motor, which is attached to the fingertip of the controller glove that the user is wearing to control the robotic hand (Figure 12). After receiving the signals, the servo rotates according to the pressure frequencies received on the force pressure sensor, making the user feel those sensations. Therefore, if the sensor touches something lightly, the servo rotates just a few degrees giving a lighter sensation. When it is pressed with maximum frequency, the servo rotates a full 180 degrees to provide a much harder sensation (Figure 13).
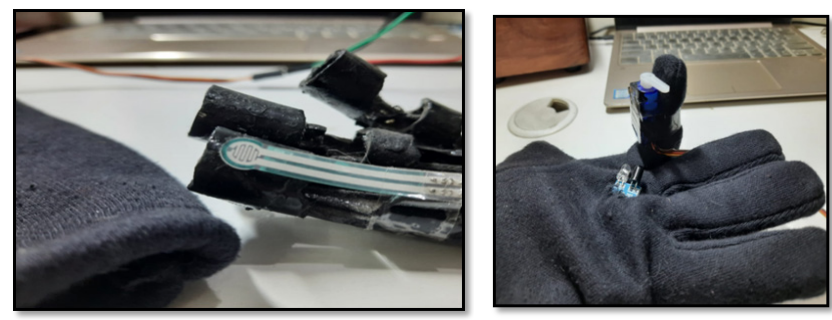

Figure 12: Force pressure sensor attached to the robotic hand.

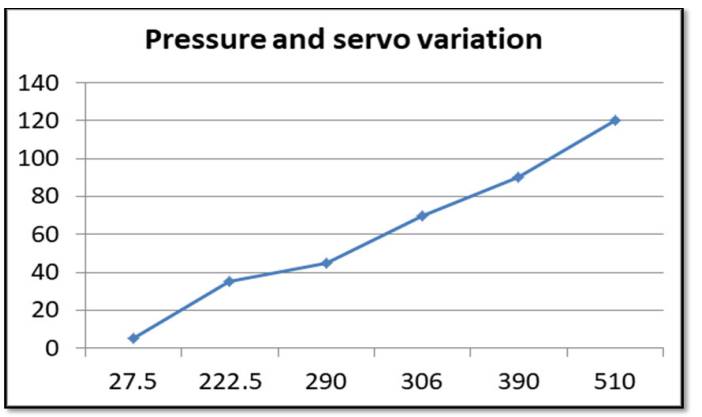

Figure 13: Pressure frequencies received by the force pressure sensor, which shows how the angle of servo increases, with the increase in pressure on the force pressure sensor

\section{Facial Expression Detection:}

This research could also assist patients with weak arm strength such as patients with Amyotrophic lateral sclerosis, stroke patients, etc. For such patients, a technology to control the robot with facial expressions, so that they do not have to use their hands, has also been developed. To serve this purpose, eyeglasses have been used to detect facial expressions. Infrared sensors, attached to the front part of the eyeglasses, detect the closing eyelid motion and send the signals to the servos, which then move the fingers of the robotic hand. 
For this, now, when the user usually closed their eyes, the sensor doesn't receive any signal. Still, when the user intentionally closes their eyes harder, the sensor senses the motion due to the harder blink and a slight movement of the eyeglass (due to the muscle movement), making the servos move to make the fingers bend. Infrared sensors placed on either side of the eyeglasses detect the motion of the user's mouth to cause action in the robotic hand (Figure 14). So, with the movement of jaw and smile, the user can rotate the wrist when they move their jaw and bend the elbow of the robotic hand with a smile on their face which would be detected by the sensors. This is also an exciting way to bring a smile to the user's face (Table 1).
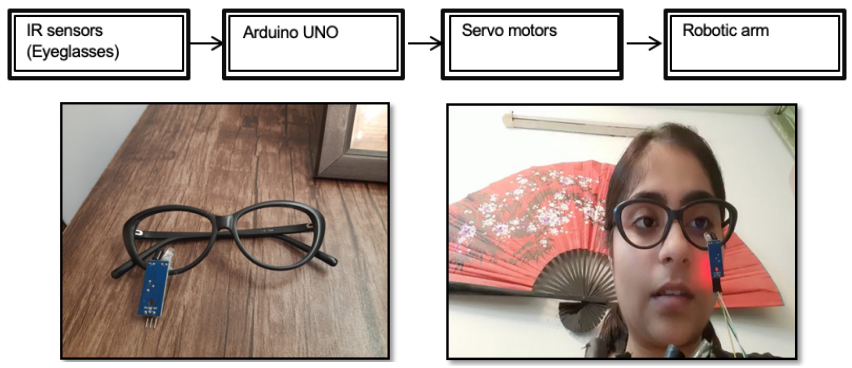

Figure 14: Smart Eyeglasses

Table 1: Commands for the Control of the Robotic Arm.

\begin{tabular}{|c|l|}
\hline Actions & Facial Expressions \\
\hline 1. Bending Fingers & Eye blink \\
\hline 2. Wrist rotation & Moving jaw \\
\hline 3. Elbow rotation & Smiling \\
\hline
\end{tabular}

\section{Pneumatic Muscles:}

The elbow movement can also be controlled by Pneumatic muscles (Mckibben muscles). Each muscle (5 g) can lift 350 g, about 70 times its weight (Figure 15; Table 2). The muscle that this project includes is $20 \mathrm{~cm}$ long and at 2 bar pressure, contracts by $1 / 7$ th of its length (Figure 16). According to the requirement, several muscles can be used to hold on to extra weight by using them as actuators. These muscles are made with a latex tourniquet tube and a nylon mesh.

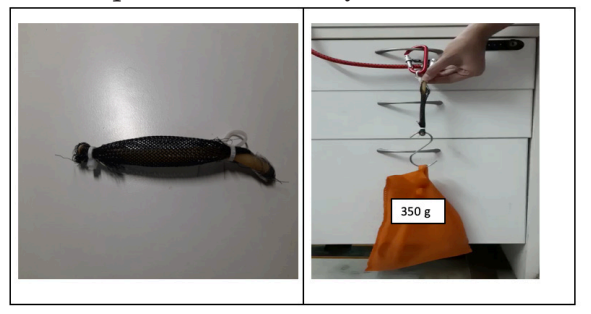

Figure 15: Smart Eyeglasses.

\section{Aplication as a bionic or a rehabilitation dervice:}

By wearing the robotic hand, the device can act as an assistive rehab device, where the user needs to wear the controller glove on the same hand as the device (Figure 17). With a slight motion of the bending finger/fingers, the user can control the robotic hand. This device could also serve as an inexpensive bionic hand. For this, the user needs to control the robotic arm with the other hand while he or she wears the device on the other (Figure 18).

Table 2: Experimental parameters.

\begin{tabular}{ccc}
\hline S.No & WEIGHT $(\mathbf{g})$ & OBSERVATION \\
\hline 1. & 100 & Lifts up \\
2. & 150 & Lifts up \\
3. & 250 & Lifts up \\
4. & 350 & Lifts up \\
5. & 400 & The tube bursts \\
\hline
\end{tabular}



Figure 16: Length variations of pneumatic muscle in response to pressure The graph shows how the muscle contracts and decreases in length with increase in pressure.
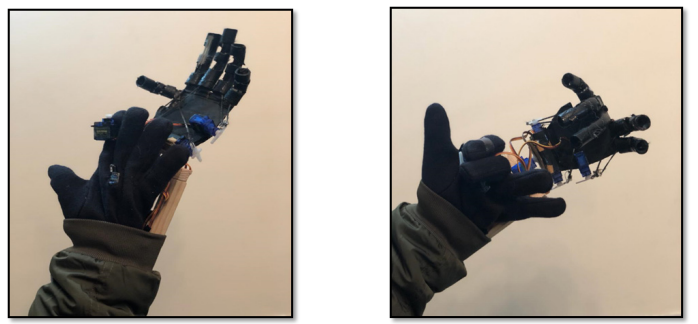

Figure 17: Application as a rehab assistant.



Figure 18: Application as a bionic arm.

\section{Conclusion}

In this research, an affordable robotic arm has been designed and developed. The mechanism used is an innovative and inexpensive version of robotic arms. Unlike high-end robotic arms that cost about $\$ 35,000$, this project costs under $\$ 100$. This research also shows how a sense of touch to the robots (haptic technology) can be added. The study demonstrates that by using different technologies through sensors, the expensive technology can be replaced. However, further research needs to be done in the future to make it fully efficient and stable. 


\section{Acknowledgements}

Without my parent's help and encouragement, this idea would not have been possible. They guided me for eight months. During this journey, they generously funded this project and gave their time, love, and support. I would also like to express my gratitude towards my schoolteachers, who held on to me with their time and patience and helped me whenever possible.

\section{References}

1. Shaikh, A.S., Panchal;, M., Maurya;, H., Shaikh;, F., Shaikh;, N., Surwadkar;, T., Juned;, M., Ashfaque, M., 2020. Multipurpose Robotic Arm. Int. J. Res. Appl. Sci. Eng. Technol. 8, 1635-1639.

2. Cattin, E., Roccella, S., Vitiello, N., Sardellitti, I., Artemiadis, P.K., Vacalebri, P., Vecchi, F., Carrozza, M.C., Kyriakopoulos, K.J., Dario, P., 2008. Design and development of a novel robotic platform for neuro-robotics applications: The NEURobotics ARM (NEURARM). Adv. Robot. 22,3-37. https://doi.org/10.1163/156855308X291827

3. Kafuko, M., Singh, I., Wanyama, T., 2015. Design of a Robotic Arm for Teaching Integrated Design. Proc. Can. Eng. Educ. Assoc. 1-5. https://doi.org/10.24908/pceea.v0i0.5827

4. Changela, B., Kataria, A., 2020. Real-Time Robotic Arm Control using Static Hand Gestures: An Approach towards the Natural Human Machine Interaction. GRD Journals-Global Res. Dev. J. Eng. 5, 8-15.

5.Patel, R., 2020. Brain Wave Controlled Robotic Arm for Paralytic and Physically Impaired Patients. Int. J. Res. Appl. Sci. Eng. Technol.8,1515-1520.https://doi.org/10.22214/ijraset.2020.30536

6. Ching-Ping Chou, \& Hannaford, B. (1996). Measurement and modeling of McKibben pneumatic artificial muscles. IEEE Transactions On Robotics And Automation, 12(1), 90-102. doi: $10.1109 / 70.481753$

\section{Author}

Vrinda Anand is a twelfth grader attending Amity International School Vasundhara 1. She is interested in mechanical engineering and plans to pursue higher studies in mechanical engineering. 